\title{
Incidental Appendectomy
}

National Cancer Institute

\section{Source}

National Cancer Institute. Incidental Appendectomy. NCI Thesaurus. Code C51888.

Surgical removal of the vermiform appendix, performed incidentally to another primary surgical procedure. 